\title{
Caracterización de salud reproductiva en perros
}

\author{
Characterization of reproductive health in dogs \\ Alfonso Sánchez Riquelme ${ }^{1,2}$, Carolina Fierro Herrera ${ }^{1}$, Francisca Troya González ${ }^{1}$, \\ Mariela Pfeffer Pfeffer ${ }^{1}$
}

\section{Resumen}

Considerando la salud reproductiva como componente importante del bienestar animal en perros, el propósito de este estudio fue caracterizar y comparar algunos parámetros de salud reproductiva en 30 perros enteros Bulldog Inglés, clínicamente sanos, distribuidos en dos grupos etarios: A, 1-4 años $(n=15)$ y B: $\geq 5$ años $(n=15)$. La evaluación se realizó a través de un examen físico reproductivo y examen ultrasonográfico testicular y prostático. Las edades promedio de los grupos A y B fueron de $2.19 \pm 1.08$ años y $7.31 \pm 3.12$ años, respectivamente. El 16.6\% del total de los ejemplares presentó prolapso uretral sin diferencias por grupo. Las frecuencias de asimetría y flacidez testicular fueron de 0.06 para ambos grupos. En la evaluación ultrasonográfica se observó una mayor frecuencia de alteraciones del parénquima testicular, especialmente heterogeneidad y presencia de focos anecoicos en el Grupo B (0.4) en comparación con el Grupo A (0.06) $(\mathrm{p}<0.05)$. En próstata, en el grupo B se observó alta frecuencia de anomalías de ecotextura: (0.8) y presencia de focos anecoicos (0.66), respecto del grupo A con $0.13 \mathrm{y}$ 0.66 respectivamente $(\mathrm{p}<0.05)$. El diámetro prostático arrojó valores de $3.06 \pm 0.45 \mathrm{~cm} \mathrm{y}$ $4.27 \pm 0.37 \mathrm{~cm}$ en los grupos A y B, respectivamente $(\mathrm{p}<0.05)$. Así mismo, se observó prostatomegalia en el $80 \%$ de los ejemplares $\geq 5$ años y solo en $20 \%$ en el grupo de perros más jóvenes $(\mathrm{p}<0.05)$.

Palabras clave: salud reproductiva; caninos; próstata; testículo; ultrasonido

\footnotetext{
${ }^{1}$ Núcleo de Investigaciones Aplicadas en Ciencias Veterinarias y Agronómicas, Universidad de Las Américas, Campus Los Castaños, Viña del Mar, Chile

${ }^{2}$ E-mail: asanchez@udla.cl/www.udla.cl
}

Trabajo financiado por el proyecto interno 2018028 de la Dirección de Investigación de la Universidad de Las Américas (UDLA)

Recibido: 12 de marzo de 2019

Aceptado para publicación: 25 de agosto de 2019 
Considering reproductive health as an important component of animal welfare in dogs, the purpose of this study was to characterize and compare some reproductive health parameters in 30 intact English Bulldog, clinically healthy, distributed in two age groups: $A, 1-4$ years $(n=15)$ and $B: \geq 5$ years $(n=15)$. The evaluation was carried out through a reproductive physical examination and testicular and prostate ultrasonographic examination. The average age of groups A and B was $2.19 \pm 1.08$ years and 7.31 \pm 3.12 years, respectively. In total, $16.6 \%$ of the specimens presented urethral prolapse without differences by age group. The frequencies of asymmetry and testicular flaccidity were 0.06 in both groups. The ultrasonographic evaluation showed a higher frequency of alterations of the testicular parenchyma, especially heterogeneity and presence of anechoic foci in Group B $(0.4)$ than in Group A $(0.06)(\mathrm{p}<0.05)$. In prostate, high frequency of ecotexture anomalies was observed $(0.8)$ and presence of anechoic foci $(0.66)$ in group $B$ with respect to 0.13 and 0.66 group $A$, respectively $(p<0.05)$. The prostate diameter had values of $3.06 \pm 0.45 \mathrm{~cm}$ and $4.27 \pm 0.37 \mathrm{~cm}$ in groups $A$ and $B$, respectively $(p<0.05)$. It should be noted that prostatomegaly was observed in $80 \%$ of the specimens $\geq 5$ years and only in $20 \%$ of the younger dogs $(\mathrm{p}<0.05)$.

Key words: reproductive health; dogs; prostate; testicle; ultrasound

\section{INTRODUCCIÓN}

En medicina humana, la salud reproductiva de la población se entiende como un estado general de bienestar físico, mental y social, y no de mera ausencia de enfermedades o dolencias, en todos los aspectos relacionados con el sistema reproductivo y sus funciones y procesos (OMS, 2018). Por su parte, la salud reproductiva en medicina veterinaria ha sido destacada, principalmente, en términos de optimizar la salud de los rebaños con el objeto de mejorar el rendimiento de la fertilidad en especies de abasto (Hafez E y Hafez B, 2002). Sin embargo, en animales de compañía el enfoque tradicional ha sido más bien reactivo y abordado esencialmente desde una perspectiva de la anormalidad y su tratamiento. Indudablemente, en el caso de especies de interés afectivo, la situación de salud es de tipo individual, de allí que resulta innovador proponer y socializar el concepto de salud reproductiva, particularmente en una dimensión preventiva, con el fin de complementar lo declarado en cuanto a bienestar animal y tenencia responsable (Sánchez, 2018).
En el macho canino (Canis lupus familiaris) se describen distintos procesos patológicos que se asocian con la presencia y funcionalidad de los testículos. Estos tienen por función la producción de espermatozoides y de hormonas reproductivas; sin embargo, trastornos metabólicos, degenerativos, neoplásicos o infecciosos pueden afectar a las gónadas generando alteraciones de la salud (Root Kustritz, 2014). La presencia de neoplasias testiculares en perros es común, describiéndose prevalencias cercanas al $30 \%$ en pacientes geriátricos (Grieco et al., 2008). Además, el perro se caracteriza por poseer próstata como única glándula sexual accesoria, la cual fisiológicamente es dependiente de la testosterona testicular (Christensen, 2018). Las enfermedades de la próstata son comunes en perros mayores de 6 años, no castrados, destacando que un alto porcentaje de estas patologías poseen características subclínicas, las que comúnmente son reportadas como hallazgos en la exploración ultrasonográfica (Smith, 2008; Polisca et al., 2016). 
La evaluación de los órganos reproductivos del perro se realiza mediante examen clínico y se complementa con ultrasonido, considerando que la ultrasonografía permite la evaluación precisa de las características del parénquima y de la arquitectura interna de dichas estructuras (Gumbsch et al., 2002; Christensen, 2018).

Para el propósito del presente estudio, se postula la hipótesis de que la salud reproductiva de perros enteros de cinco o más años presentaría más alteraciones subclínicas respecto de animales más jóvenes de la misma raza. En el presente estudio se caracteriza y comparan algunos parámetros de salud reproductiva en perros enteros de dos grupos etarios a través de un examen físico reproductivo y exámenes ultrasonográfico testicular y prostático.

\section{Materiales y Métodos}

Mediante un muestreo por conveniencia y con el consentimiento de sus propietarios, se examinaron 30 perros de raza Bulldog Inglés, clínicamente sanos (examen clínico general) y mayores de un año. El criterio de inclusión consideró la presencia de ambos testículos en el escroto y la ausencia de signos evidentes de alteración prostática. El estudio se realizó entre enero de 2018 y febrero de 2019 y los ejemplares fueron evaluados en el Centro Veterinario de la Universidad de Las Américas, sede Viña del Mar (Chile). La información recolectada incluyó edad, peso vivo e historial reproductivo. Se conformaron dos grupos etarios: Grupo A: 1 a 4 años $(n=15)$ y Grupo B: e»5 años ( $n=15)$. El protocolo de trabajo con animales fue aprobado por el Comité de Ética Científica de la Universidad de Las Américas (UDLA).

El examen físico reproductivo consideró palpación e inspección de pene y prepucio, inspección y medición de perímetro escrotal con pie de metro (Figura 1), palpación testicular y palpación de epidídimos
(Root Kustritz, 2014). Los exámenes ultrasonográficos se realizaron por el mismo operador con el paciente en estación, sin necesidad de sedación, utilizando un equipo Mindray M5Vet con transductor microconvexo de 5-8 MHz. La evaluación testicular se realizó en los planos longitudinal y transversal, y consideró ecogenicidad del parénquima, visualización de mediastino y presencia o ausencia de estructuras anecoicas y/o hiperecoicas (Gumbsch et al., 2002). La evaluación prostática se realizó en los planos longitudinal y transversal, y consideró situación, tamaño, forma, simetría, estructura capsular y ecotextura del parénquima (homogéneo, heterogéneo hipoecoico o heterogéneo hiperecoico), contorno (conservado o irregular), presencia y cantidad de focos anecoicos e hiperecoicos. Además, se evaluó el diámetro glandular en el plano transversal (Ruel et al., 1998; Atalan et al., 1999).

Con las variables continuas se realizó estadística descriptiva, calculando media y desviación estándar y para variables categóricas se estimaron las frecuencias de cada categoría. Para variables continuas se chequearon los supuestos del test de Student, normalidad y homogeneidad de la varianza. Para las variables categóricas se utilizó el test no paramétrico Mann-Whitney. Todos los análisis estadísticos fueron realizados con el programa estadístico STATA 14, considerando un $95 \%$ de confianza $(\mathrm{p}<0.05)$.

\section{Resultados}

El rango de edad en la muestra total fluctuó entre 1 y 11 años. La edad promedio fue de $2.18 \pm 1.08$ años en el Grupo A y de $7.31 \pm 3.12$ años en el Grupo B, respectivamente $(\mathrm{p}<0.05)$. Los pesos promedios resultaron similares: $26.4 \pm 3.9 \mathrm{~kg}$ en el Grupo A y $27.0 \pm 3.4 \mathrm{~kg}$ en el Grupo B. La anamnesis arrojó que solo el 30\% de los canes había sido utilizado con fines reproductivos, especialmente en el grupo de perros $\geq 5$ años (6/30). Según los antecedentes recogidos, en todos 


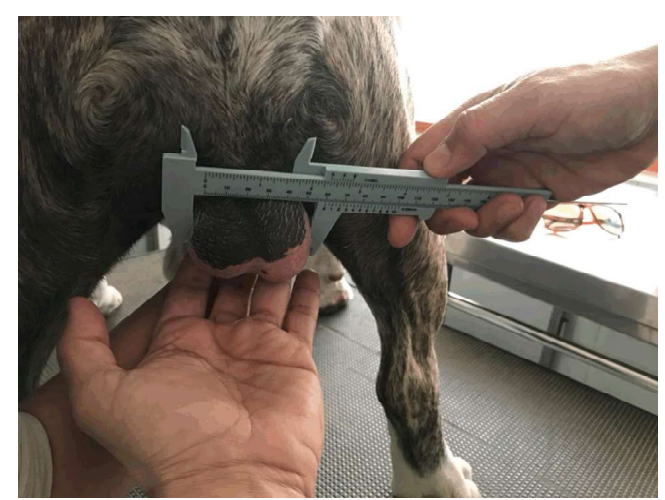

Figura 1. Medición de perimetro escrotal en un perro Bulldog inglés de 2 años

los casos se practicó extracción de semen para inseminación artificial y hubo inscripción de camadas, sin que se haya podido precisar el tamaño de estas. La evaluación física de pene y prepucio indicó que el 16.6\% (5/30) de los ejemplares presentaron prolapso uretral (Figura 2), correspondiendo 2 casos al Grupo A y 3 casos al Grupo B.

En el examen físico reproductivo se registraron mayores frecuencias de anomalías testiculares. Entre estas, asimetría (8/15) y tono disminuido (11/15) en el grupo de perros $\geq 5$ años con relación al grupo de ejemplares más jóvenes (1/15) y (1/15), respectivamen-

Cuadro 1. Frecuencias de alteraciones prostáticas observadas mediante ultrasonografía en perros de raza Bulldog Inglés de dos grupos etarios (Viña del Mar, Chile)

\begin{tabular}{lcc}
\hline & $\begin{array}{c}1-4 \text { años } \\
(\mathrm{n}=15)\end{array}$ & $\begin{array}{c}\geq 5 \text { años } \\
(\mathrm{n}=15)\end{array}$ \\
\hline Anomalías de & $0.13^{\mathrm{a}}$ & $0.80^{\mathrm{b}}$ \\
ecotextura & $0.13^{\mathrm{a}}$ & $0.73^{\mathrm{b}}$ \\
Asimetría lobular & $0.60^{\mathrm{b}}$ \\
Contorno irregular & $0^{\mathrm{a}}$ & $0.0 .66^{\mathrm{b}}$ \\
Focos anecoicos & $0.06^{\mathrm{a}}$ & $0.8^{\mathrm{b}}$ \\
Aumento de tamaño & $0.2^{\mathrm{a}}$ & $0.8^{\mathrm{b}}$ \\
a,b Superíndices diferentes indican diferencias \\
\multicolumn{2}{l}{ significativas ( $\mathrm{p}<0.05)$}
\end{tabular}

te $(p<0.05)$. El perímetro escrotal no arrojó diferencias entre grupos, registrándose valores de $5.11 \pm 0.73$ y $4.98 \pm 0.67$ en los grupos A y B, respectivamente. En la evaluación ultrasonográfica se pudo observar una mayor frecuencia de alteraciones del parénquima testicular, especialmente heterogeneidad y presencia de focos anecoicos en los perros $\geq 5$ años (6/15) (Figura 3) versus el grupo de $1-4$ años $(1 / 15)(\mathrm{p}<0.05)$. Por otro lado, en la evaluación física y ecográfica no se observaron alteraciones epididimarias en los grupos en estudio.

En la evaluación de la glándula prostática (Cuadro 1) destacó la alta proporción de anomalías de ecotextura (12/15) y presencia de focos anecoicos (10/15) en el grupo de perros e»5 años (Figura 4), respecto del grupo de animales más jóvenes $(2 / 15$ y $1 / 15$, respectivamente, $p<0.05$ ). El diámetro prostático arrojó valores de $3.06 \pm 0.45 \mathrm{~cm} \mathrm{y}$ $4.27 \pm 0.37 \mathrm{~cm}$ en los grupos A y B, respectivamente $(\mathrm{p}<0.05)$, lo cual se puede comparar con el valor de referencia de $3.44 \pm 0.72$ $\mathrm{cm}$, dado por Miguel de Priego (2007). Cabe destacar que la prostatomegalia se observó en el $80 \%$ de los ejemplares $\geq 5$ años y solo en $20 \%$ de los animales más jóvenes ( $p<0.05$ ), alcanzando un $46 \%$ en el total de la muestra.

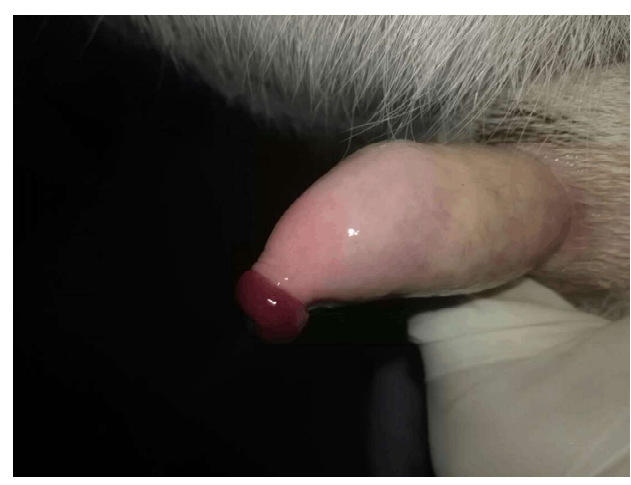

Figura 2. Presencia de prolapso uretral en un perro Bulldog inglés de 6 años 


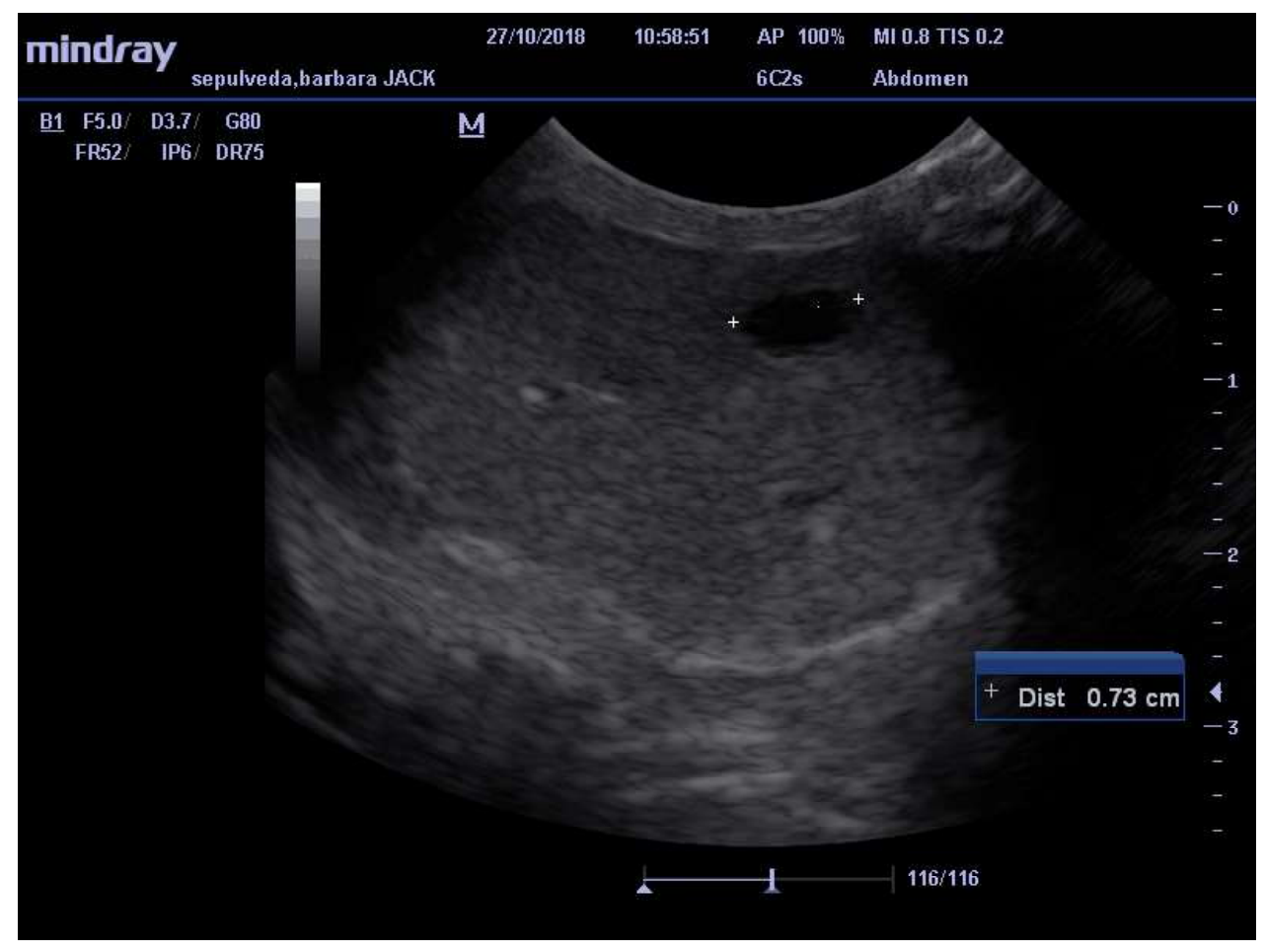

Figura 3. Imagen ecográfica, en corte longitudinal, de testículo derecho con presencia de focos anecoicos en un perro Bulldog inglés de 6 años

\section{Discusión}

En el presente estudio cabe destacar que un bajo porcentaje de los perros había sido utilizado para fines reproductivos y que los individuos con edades $\geq 5$ años que no estaban destinados a la reproducción o que habían finalizado su vida reproductiva no estaban castrados. La presencia de prolapsos uretrales en varios ejemplares llama la atención, aunque este hallazgo resultó ser incidental. Según Peña (2006) y Dumon (2009), esta es una afección rara, descrita principalmente en las razas Boston Terrier y Bulldog Inglés, y posiblemente debido al sobresfuerzo en la micción o el exceso de estímulo sexual.

En el examen físico, los testículos deben ser simétricos en tamaño y forma, de consistencia firme y deben desplazarse libremente en el escroto (Root Kustritz, 2014).
Esta situación estuvo alterada en alta proporción en el grupo de perros $\geq 5$ años, destacando una mayor presentación de flacidez y disminución de tamaño en el testículo derecho, determinando una asimetría testicular. Esto tiene relación con lo descrito por SchäferSomi (2015), quien afirma que en el examen andrológico de individuos de mayor edad se evidencian alteraciones testiculares que incluyen una disminución del parénquima gonadal, fenómeno asociado a degeneración $\mathrm{y}$ atrofia testicular.

Según Bhanmeechao et al. (2018), los cambios degenerativos en los testículos caninos son más pronunciados sobre los 9 años. Por otra parte, McEntee (1990) señala que cambios macroscópicos no serían evidentes en los grados leves a moderados de degeneración, a diferencia de lo que ocurre en los perros de mayor edad, donde se observa una disminución del tamaño testicular debido a una 


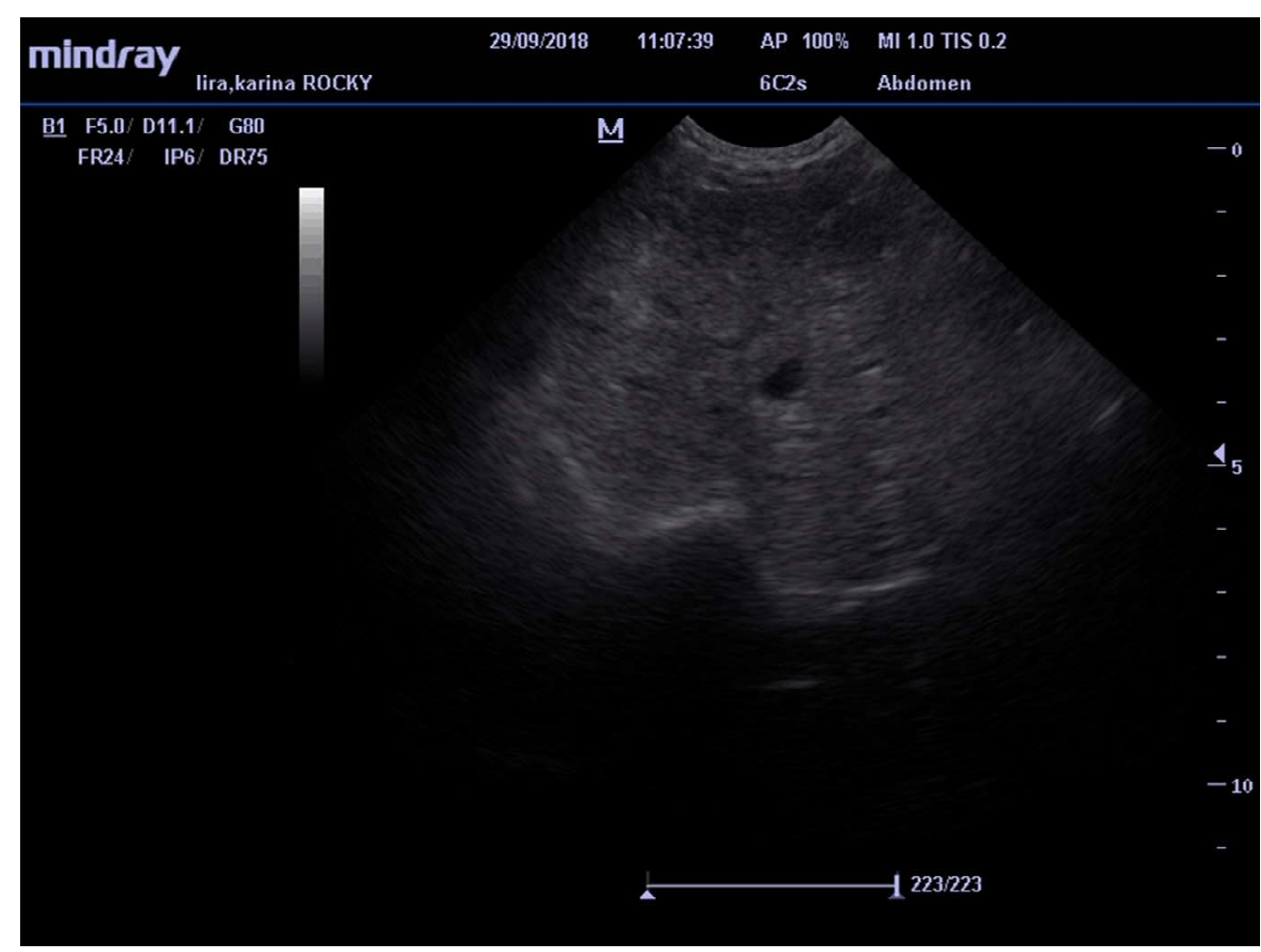

Figura 4. Imagen ecográfica de próstata, en corte transversal, con parénquima heterogéneo, presencia de focos anecoicos y contorno glandular anormal en un perro Bulldog inglés de 8 años

degeneración avanzada. Dado que los perros del Grupo B presentaron una edad promedio de 7.31 años, se les podría considerar como animales viejos (Bhanmeechao et al., 2015), las observaciones del examen físico reproductivo con el examen ultrasonográfico testicular resultan concordantes, ya que en este último se observó una mayor presencia de anomalía de ecotextura en el parénquima y de focos anecoicos en este grupo de perros. No obstante esta observación, los perímetros escrotales resultaron similares para ambos grupos, con valores considerados como normales para el peso de los ejemplares (Wooddall y Johnstone, 1988).

Cabe destacar que como criterio de inclusión en este estudio los perros no presentaban signos evidentes de compromiso prostático, tales como hematuria, disuria, constipación o tenesmo (Lévy et al., 2014).
Sin embargo, en el examen ultrasonográfico en los perros $\geq 5$ años se observó una alta proporción de alteraciones (anomalías de ecotextura, asimetría lobular, contorno irregular y presencia de focos anecoicos) y aumento de tamaño glandular, descripción que coincide con varios autores (Barsanti y Finco, 2002; Smith, 2008; Lee et al., 2011; Peña et al., 2013; Krakowski et al., 2015; Polisca et al., 2016). Respecto al aumento de tamaño prostático, cabe destacar que el $46 \%$ de casos de prostatomegalia del presente estudio se ubica entre el 33 y $84 \%$ reportado por Mantziaras et al. (2017) e Ygreda et al. (2117). Estos autores describen la prostatomegalia como la característica más frecuente en perros enteros entre 1 y 18 años. Por otra parte, Polisca et al. (2016) en un estudio retrospectivo, destacan que la edad promedio de perros con desórdenes prostáticos es de $8.6 \pm 3,2$ años, siendo $6.2 \pm$ 
2.3 la edad en que se diagnostica más comúnmente la hiperplasia prostática benigna (HPB) en pacientes asintomáticos.

De acuerdo con lo propuesto por Ygreda et al. (2017), relacionando la compatibilidad de las alteraciones ultrasonográficas con tipos de patologías específicas de la próstata, se podría considerar la presencia de HPB, la cual coincidió en cerca de un 70\% de los casos con presencia de estructuras quísticas únicas o múltiples en el parénquima glandular. Dicha condición se describe habitualmente en perros enteros mayores de 5 años (Ruel et al., 1998; Johnston et al., 2001), con una prevalencia de $90 \%$ para individuos entre 7.1 y 8 años (Christensen, 2018). Dado lo anterior, resulta interesante destacar lo planteado por Mantziaras et al. (2017), quienes señalan que la incidencia de enfermedades prostáticas ha aumentado en los últimos años debido al incremento en las expectativas de vida de los perros.

En concordancia con la hipótesis propuesta en el estudio, los perros con edades $\geq 5$ años presentaron una serie de alteraciones testiculares y prostáticas subclínicas, las cuales potencialmente podrían derivar en un detrimento del bienestar de los animales, postulando por ello la importancia de establecer pautas en salud reproductiva, a fin de mejorar la calidad de vida de las mascotas, educar a los tenedores en la conveniencia de la esterilización temprana de ejemplares sin propósitos de cría y en el caso de animales de valor reproductivo, generar recomendaciones a los médicos veterinarios, para la realización de estudios preventivos (Sánchez, 2018). En este sentido, se ha propuesto realizar exámenes prostáticos anuales a partir de los 5 años de edad en ejemplares con valor reproductivo para la detección precoz de alteraciones y comenzar los exámenes a los 6 o 7 años en el caso de animales sin valor reproductivo (Polisca et al., 2016). Por otro lado, Mantziaras et al. (2017) plantean que los exámenes preventivos con el fin de detectar tempranamente anormalidades prostáticas deberían comenzar a realizarse al cumplir el $40 \%$ de su expectativa de vida.

\section{Conclusión}

Se registró una mayor proporción de anormalidades testiculares y prostáticas en el grupo de perros $\geq 5$ años, en comparación con perros entre 1 y 4 años de edad.

\section{Literatura Citada}

1. Atalan G, Holt P, Barr J, Brown P. 1999. Ultrasonographic estimation of prostatic size in canine cadavers. Res Vet Sci 67: 7-15. doi: 10.1053/rvsc.1998.0267

2. Barsanti J, Finco D. 2002. Enfermedades prostáticas caninas. En: Ettinger S, Feldman E (eds). Tratado de medicina interna veterinaria. $6^{\circ}$ ed. España. Elsevier. p 1952-1971.

3. Bhanmeechao C, Srisuwatanasagul S, Ponglowhapan S. 2018. Age-related changes in interstitial fibrosis and germ cell degeneration of the canine testis. Reprod Domest Anim 53: 37-43. doi: 10.1111/rda.13354

4. Christensen B. 2018. Canine prostate disease. Vet Clin NAm-Small 48: 701-719.

5. Dumon C. 2009. Patología de la reproducción en la especie canina. Casos clínicos. Buenos Aires: Inter-Médica. 480 p.

6. Grieco V, Riccardi E, Greppi G, Teruzzi F, Iermano V, Finazzi $M$. 2008. Canine testicular tumours: a study on 232 dogs. J Comp Path 138: 86-89. doi: 10.1016/j.jcpa.2007.11.002

7. Gumbsch P, Gabler C, Holzmann A. 2002. Colour coded duplex sonography of the testes of dogs. Vet Rec 151: 140144. doi: 10.1136/vr.151.5.140

8. Hafez E, Hafez B. 2002. Reproducción e inseminación artificial en animales. McGraw-Hill Interamericana. México. $256 \mathrm{p}$.

9. Johnston S, Root Kustritz M, Olson P. 2001. Disorders of the canine prostate. Canine and feline theriogenology. Philadelphia: Saunders. p 337-355. 
10. Krakowski L, Wachocka A, Brodzki P, Wrona Z, Piech T, Wawron W, Chalabis-Mazurek A. 2015. Sperm quality and selected biochemical parameter of seminal fluid in dogs with benign prostatic hiperplasia. Anim Reprod Sci 160: 120-125. doi: 10.1016/ j.anireprosci.2015.07.014

11. Lee K, Shimizu J, Kishimoto M, Kadohira M, Iwasaki T, Miyake Y, Yamada K. 2011. Computed tomography of the prostate gland in apparently healthy entire dogs. J Small Anim Pract 52: 141-151. doi: 10.1111/j.17485827.2011.01041.x

12. Lévy X, Nizanski W, Von Heimendahl A, Mimouni P. 2014. Diagnosis of common prostatic conditions in dogs: an update. Reprod Domest Anim 49: 50-57. doi: 10.1111/rda.12296

13. Mantziaras G, Alonge S, Faustini M, Luvoni G 2017. Assessment of age for a preventive ultrasonographic examination of the prostate in the dog. Theriogenology 100: 114-119. doi: 10.1016/j.theriogenology.2017.06.010

14. McEntee K. 1990. Reproductive pathology of domestic mammals. San Diego, California, USA: Academic Press. $401 \mathrm{p}$.

15. Miguel de Priego C. 2007. Correlación entre el tamaño ecográfico de la glándula prostática y el riñón izquierdo en caninos. Tesis de Médico Veterinario Zootecnista. Lima, Perú: Univ. Peruana Cayetano Heredia. 16 p.

16. [OMS] Organización Mundial de la Salud. 2018. Salud reproductiva. [Internet] Disponible en: http:// www.who.int/topics/reproductive_health/es

17. Peña F. 2006. Fisiología reproductiva del macho. En: Wanke M, Gobello C. Buenos Aires: Inter-Médica. p 129-151.
18. Peña S, Chavera A, Cahua J, Perales R. 2013. Alteraciones prostáticas en caninos determinadas mediante ultrasonografía y citología por aspiración eco-guiada. Rev Inv Vet Perú 24: 207-216. doi: 10.15381/rivep.v24i2.2502

19. Polisca A, Troisi A, Fontain, E, Menchetti L, Fontbonne A. 2016. A retrospective study of canine prostatic disease from 2002 to 2009 at the Alfort Veterinary College in France. Theriogenology 85: 835-840. doi: 10.1016/ j.theriogenology.2015.10.030

20. Root Kustritz M. 2014. Applied small animal andrology. In: Animal andrology: theories and applications. Chenoweth P, Lorton S. CAB International. p 177-196.

21. Ruel Y, Barthez P, Mailles A, Begon D. 1998. Ultrasonographic evaluation of the prostate in healthy intact dogs. Vet Radiol Ultrasoun 39: 212-216.

22. Sánchez A. 2018. Salud reproductiva del macho canino. Invest Cienc Anim 2: 33-36.

23. Schäfer-Somi S. 2015. New approaches to semen improvement in dogs. Rev Bras Reprod Anim 39: 141-145.

24. Smith J. 2008. Canine prostatic disease: a review of anatomy, pathology, diagnosis, and treatment. Theriogenology 70 : 375-383. doi: $10.1016 / \mathrm{j}$.theriogenology.2008.04.039

25. Woodall P, Johnstone I. 1988. Dimentions and allometry of testes, epididymides and spermatozoa in the domestic dog (Canis familiaris). J Reprod Fertil 82: 603-609. doi: 10.1530/ jrf.0.0820603

26. Ygreda G, Grandez, Ralencia $R$. 2017. Caracterización de las alteraciones ultrasonográficas prostáticas en perros atendidos en una clínica veterinaria en Lima, Perú, entre 2010 y 2014. Rev Inv Vet Perú 28: 299-306. doi: 10.15381/ rivep.v28i2.12480 